\title{
Ability of Alere ${ }^{\mathrm{tw}}$ HIV Combo to diagnose acute HIV infection is based mainly on HIV-1 p24 antigen detection
}

\author{
Sunee Sirivichayakul, ${ }_{1}$ Tippawan Pankam, ${ }^{2}$ Supanit Pattanachaiwit, ${ }^{2}$ Kannapat Phancharoen, ${ }^{2}$ Napapat Barisri, ${ }^{2}$ \\ Supphachoke Areeyolwattana, ${ }^{2}$ Nittaya Phanuphak, ${ }^{3,4}$ Praphan Phanuphak ${ }^{1,3}$
}

\begin{abstract}
Background: Alere ${ }^{\mathrm{mm}}$ HIV Combo is the only rapid and sensitive point-of-care $4^{\text {th }}$ generation (antigen/antibody) HIV test newly available in Thailand which is advantageous of differentiating between positivity of antigen or antibody or both, especially in acute HIV infection (AHI). AHI in this study was defined by positive machine-based $4^{\text {th }}$ generation test with measurable HIV-RNA but negative $3^{\text {rd }}$ generation (IgM/IgG antibody only) tests.
\end{abstract}

Objective: To evaluate the performance characteristics of Alere ${ }^{\mathrm{Tm}}$ HIV Combo.

Methods: Fifty stored plasma samples of subjects diagnosed with AHI were used to evaluate Alere ${ }^{\mathrm{TM}}$ HIV Combo.

Results: Of the 50 AHI samples, Alere ${ }^{\mathrm{mm}}$ HIV Combo was positive in 37 (74\%): 5 with antibody positive only (R1), 26 with p24 antigen positive only (R2), and 6 with both p24 antigen and antibody positive (R3). Mean sample/cut-off (S/ $\mathrm{CO})$ ratios from machine-based $4^{\text {th }}$ generation test of R1, R2 and R3 were $21.55,148.38$ and 72.97 respectively as compared to 7.57 for the 13 non-reactive (NR) samples. The corresponding median $\log _{10}$ HIV-RNA of NR, R1, R2 and R3 were 5.59, 5.86, 7.00 and 6.61 copies/mL respectively. R2 and R3 had significantly higher S/CO and HIV-RNA than NR and R1. Alere ${ }^{\mathrm{Tm}}$ HIV Combo detected mainly p24 antigen in AHI as seen in 32/50 (64\%) subjects and could detect $11 / 50(22 \%)$ antibody in AHI samples which were missed by the two $3^{\text {rd }}$ generation HIV tests used in our testing algorithm.

Conclusions: Alere ${ }^{\mathrm{Tm}}$ HIV Combo is the ideal screening test in a setting with high AHI where machine-based $4^{\text {th }}$ generation test is not available.

Key words: rapid $4^{\text {th }}$ generation HIV test, acute HIV infection, p24 antigen, Alere ${ }^{\mathrm{Tm}}$ HIV Combo, Rapid distinguishable antigen-antibody HIV test

\footnotetext{
From:

Department of Medicine, Faculty of Medicine, Chulalongkorn University, Bangkok, Thailand

2 Thai Red Cross Anonymous Clinic Laboratory,

Thai Red Cross AIDS Research Centre, Bangkok, Thailand

${ }^{3}$ Institute of HIV Research and Innovation (IHRI), Bangkok, Thailand

${ }^{4}$ Center of Excellence in Transgender Health (CETH),

Chulalongkorn University, Bangkok, Thailand
}

\section{Introduction}

HIV testing is the limiting gateway for antiretroviral therapy (ART) and HIV prevention, particularly pre-exposure prophylaxis (PrEP). Early and frequent HIV testing, immediate ART and large-scale PrEP implementation have proved to collectively reduce the number of new HIV infections in big cities throughout the world. ${ }^{1-3}$ In addition to addressing psychosocial and structural barriers through innovative HIV testing service delivery models, HIV test kit itself also plays

\author{
Corresponding author: \\ Sunee Sirivichayakul \\ 1873 Rama IV Road, Pathumwan, Bangkok 10330, Thailand \\ E-mail: Sunee.S@chula.ac.th
}

an important role in achieving early diagnosis of HIV infection. Rapid HIV test (RT) with high sensitivity is needed most in busy laboratories as well as for mobile or community-based/community-led HIV testing. ${ }^{4-6}$

Many RT kits are prequalified by the World Health Organization (WHO) but not all are available in each country. ${ }^{7}$ For example, in Thailand, 14 out of the 17 WHO-prequalified RT are licensed for sale and only 12 are available on the market. ${ }^{8}$ 
In general, fourth generation (antigen/antibody) RT is preferable to second or third generation antibody rapid tests due to its added ability to detect HIV-1 p24 antigen in acute or early HIV infection when antibody may not yet have been formed. ${ }^{9}$ Determine HIV-1/2 Ag/Ab Combo test was the first $4^{\text {th }}$ generation RT available in the market. ${ }^{10}$ However, it has since been replaced by a newer version, Alere ${ }^{\mathrm{Tm}}$ HIV Combo, which is more sensitive in detecting p24 antigen in subjects with acute or early HIV infection. ${ }^{11}$ Alere ${ }^{\mathrm{Tm}}$ HIV Combo is the only $4^{\text {th }}$ generation RT recently available in Thailand. ${ }^{8}$ The test is a qualitative, visually read immunoassay for the simultaneous detection of HIV Type 1 (HIV-1) p24 antigen, and antibodies to HIV Types 1 and 2 (HIV-1 and HIV-2) in human serum, plasma and finger stick or venipuncture whole blood. ${ }^{8}$ It is intended for use as point-of-care diagnostic test for HIV-1 and/ or HIV-2 infection, including acute HIV-1 infection. ${ }^{11-15}$

Alere $^{\mathrm{Tx}}$ HIV Combo has been carefully evaluated in the field in both HIV-1 and HIV-2 infected populations and in both acute and established HIV-1 infections. ${ }^{11,13-15}$ In seroconversion panels, sensitivity of Alere ${ }^{\mathrm{ms}}$ HIV Combo fell between machine-based $4^{\text {th }}$ generation test and $3^{\text {rd }}$ generation $(\operatorname{IgM} /$ IgG antibody) test. ${ }^{15}$ In this report, we intended to compare the sensitivity of Alere ${ }^{\mathrm{mm}}$ HIV Combo with machine-based $4^{\text {th }}$ generation test in a panel of well-characterized plasma samples from acutely HIV infected Thai individuals who were positive on machine-based $4^{\text {th }}$ generation and HIV-RNA tests but negative antibody-alone test.

\section{Materials and Methods \\ Study population}

Prior to the initiation of this evaluation, Alere ${ }^{\mathrm{Tm}}$ HIV COMBO was found to have $100 \%$ sensitivity and specificity when evaluated against 100 confirmed HIV positive and 100 HIV negative plasma samples from the Thai Red Cross Anonymous Clinic (TRC-AC) in Bangkok. HIV positivity was defined by positive Abbott machine-based $4^{\text {th }}$ generation ARCHITECT HIV Ag/Ab Combo screening assay (Abbott Diagnostics, Lake Forest, IL, USA), confirmed by two positive $3^{\text {rd }}$ generation antibody tests, Alere Determine HIV-1/2 (Alere Medical Co., Ltd. Chiba, Japan) and gel particle agglutination (Fujirebio Diagnostics, Inc., Tokyo, Japan), according to the routine HIV testing algorithm used at the Clinic. ${ }^{16}$ HIV negativity was defined by negative $4^{\text {th }}$ generation ARCHITECT $\mathrm{HIV} \mathrm{Ag} / \mathrm{Ab}$ Combo assay and confirmed by negative pooled qualitative HIV RNA assay (Aptima).

Evaluation of Alere ${ }^{\mathrm{Tm}}$ HIV Combo was performed with a set of 50 stored plasma samples from individuals with acute HIV infection (AHI). Samples were collected between January 2014 to March 2015, from a large AHI cohort established at the TRC-AC since 2009, with well-defined clinical, serological and viral characteristics. ${ }^{17}$ All study samples were decoded with removal of all demographic information before the researchers accessed them. AHI in this set of plasma samples was defined by positive machine-based $4^{\text {th }}$ generation screening test but negative by both confirmatory antibody tests whereas both qualitative HIV-RNA assay (Aptima, Gen-Probe
Inc, San Diego, CA, U.S.A.) and quantitative HIV-RNA assay (Abbott RealTime HIV-1 Amplification Reagent Kit, Abbott Molecular Inc. IL, USA) were positive. Sample/cut-off (S/CO) ratio of the screening test and the HIV-RNA viral load (VL) of each sample were used for group analysis. All subjects in the AHI cohort have signed the study consent form, including the consent to use their stored biological specimens for future research purposes as approved by the Institutional Review Board of Faculty of Medicine, Chulalongkorn University.

\section{Alere $^{\text {Tx }}$ HIV Combo testing}

Principle of the assay is immunochromatography. The test was performed according to the manufacturer's protocol. ${ }^{18}$ In brief, 50 ul plasma sample is applied on the sample pad, if HIV-1 p24 antigen is present, it will bind to the biotinylated anti-p24 antibodies and the selenium colloid-conjugate, coated with anti-p24 mouse monoclonal antibody from the Sample Pad to form a complex which will be captured by immobilized avidin at the Upper Test Area (labeled "Ag") to form a single pink/red "Ag" line. If antibodies to HIV-1 and/ or HIV-2 are present, the antibodies will bind to the selenium colloid-conjugate, coated with recombinant HIV-1, HIV2 and HIV-1 group $\mathrm{O}$ antigens and synthetic HIV-2 peptide from the Conjugate Pad, which will then be captured by immobilized recombinant $\mathrm{HIV}-1 / \mathrm{HIV}-1$ group $\mathrm{O}$ antigens and synthetic HIV-1/HIV-2 peptides at the Lower Test Area (labeled "Ab") to form a single pink/red "Ab" line. The results of individual samples were read by three independent medical technologists. Any discordant visual readings were resolved by two out of the three medical technologists. Samples with discordant results between machine-based $4^{\text {th }}$ generation test and Alere ${ }^{\mathrm{TM}}$ HIV Combo were re-tested in duplicate with both procedures. Sensitivity and specificity of Alere ${ }^{\mathrm{Tm}} \mathrm{HIV}$ Combo along with $95 \%$ confidence intervals (CI) were calculated. Statistical analysis of S/CO ratio and HIV-RNA $\left(\log _{10}\right.$ copies $\left./ \mathrm{mL}\right)$ were calculated by Kruskal-Wallis Test and ANOVA, respectively.

\section{Results \\ HIV testing performance of Alere ${ }^{\mathrm{Tn}} \mathrm{HIV}$ Combo in AHI and its correlation with S/CO ratio}

Alere $^{\text {ts }}$ HIV Combo could detect 37 out of 50 AHI samples, a sensitivity of $74 \%$ (95\% CI $61.41 \%$ to $86.59 \%)$. We further categorized the 37 Alere $^{\mathrm{mm}}$ HIV Combo reactive (R) samples into: antibody positive only ( $\mathrm{R} 1, \mathrm{~N}=5), \mathrm{p} 24$ antigen positive only ( $2, \mathrm{~N}=26)$, and both $\mathrm{p} 24$ antigen and antibody positive ( $\mathrm{R} 3, \mathrm{~N}=6)$. Compared to the reactive $(\mathrm{R})$ samples, Alere ${ }^{\mathrm{TM}}$ HIV Combo non-reactive samples (NR, $\mathrm{N}=13$ ) had significantly lower mean (SD) S/CO ratio 7.57 (6.95) vs. 119.01 (121.98), $p \leq 0.001$. Mean (SD) S/CO ratio of R1, R2 and R3 were 21.55 (27.28), 148.38 (132.84) and 72.97 (42.24), respectively (Table 1 and Figure 1). R2 and R3 had significantly higher S/CO than $\mathrm{R} 1(p=0.01$ and $p=0.03$, respectively) and NR (both $p<0.001$ ). There was no discordant result among the three medical technologists assigned to separately read each of the Alere ${ }^{\mathrm{TM}}$ HIV Combo strips. 
Table 1. Mean S/CO ratio of $4^{\text {th }}$ generation ARCHITECT HIV Ag/Ab Combo assay and median $\log _{10}$ HIV-RNA of designated groups of 50 acute HIV samples as classified by Alere ${ }^{\mathrm{rm}}$ HIV Combo test results

\begin{tabular}{|lcc|}
\hline \multicolumn{1}{|c|}{$\begin{array}{c}\text { Acute HIV samples }(\mathbf{N}) \text { classified by } \\
\text { Alere }^{\mathrm{Tn}} \text { HIV Combo test results }\end{array}$} & $\begin{array}{c}\text { Mean S/CO ratio of } \\
\mathbf{4}^{\text {th }} \text { generation IA test }\end{array}$ & $\begin{array}{c}\text { Median HIV-RNA } \\
\left(\log _{10} \text { copies/mL) }\right.\end{array}$ \\
\hline Non-reactive, NR (13) & $7.57(\mathrm{a})$ & $5.59(\mathrm{a})$ \\
\hline Antibody positive, R1 (5) & $21.55(\mathrm{~b})$ & $5.86(\beta)$ \\
\hline p24 antigen positive, R2 (26) & $148.38(\mathrm{c})$ & $7.00(\lambda)$ \\
\hline p24 antigen and antibody positive, R3 (6) & $72.97(\mathrm{~d})$ & $6.61(\mu)$ \\
\hline
\end{tabular}

(a) NR was not significantly different from R1 $(p>0.05)$ but significantly different from R2 $(p<0.001)$ and R3 $(p=0.001)$.

(b) R1 was not significantly different from NR $(p>0.05)$ but significantly different from R2 $(p=0.01)$ and R3 ( $p=0.03)$.

(c) R2 was not significantly different from R3 $(p>0.05)$ but significantly different from NR $(p<0.001)$ and R1 $(p=0.01)$.

(d) R3 was not significantly different from R2 $(p>0.05)$ but significantly different from NR $(p=0.001)$ and R1 $(p=0.03)$.

(a) NR was not significantly different from R1 $(p>0.05)$ but significantly different from R2 $(p<0.001)$ and R3 $(p=0.01)$.

( $\beta$ ) R1 was not significantly different from NR $(p>0.05)$ and R3 $(p=0.08)$ but significantly different from R2 $(p=0.003)$.

$(\lambda)$ R2 was not significantly different from R3 $(p>0.05)$ but significantly different from NR $(p<0.001)$ and R1 $(p=0.003)$.

$(\mu)$ R3 was not significantly different from R1 $(p=0.08)$ and R2 $(p>0.05)$ but significantly different from NR $(p=0.01)$.

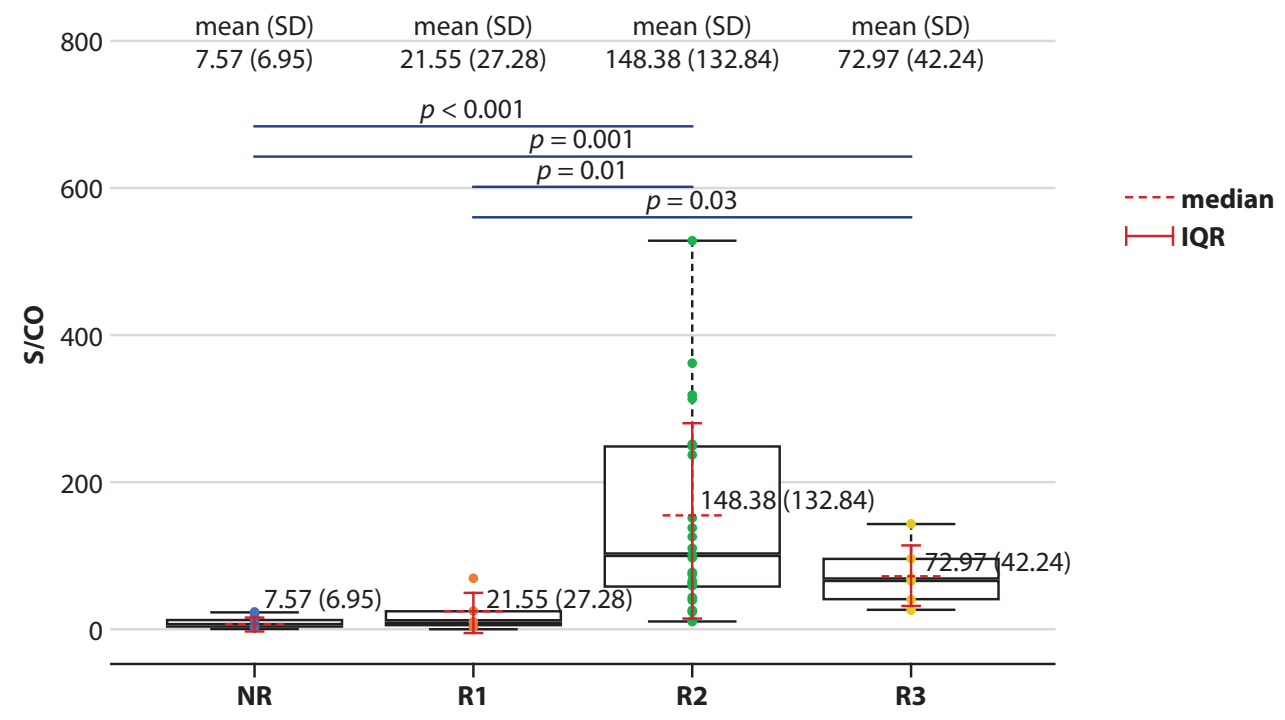

Figure 1. Boxed plot illustration of S/CO with mean (SD) in NR (non-reactive), R1(antibody positive only), R2 (p24 antigen positive only) and R3 (both p24 antigen and antibody positive)

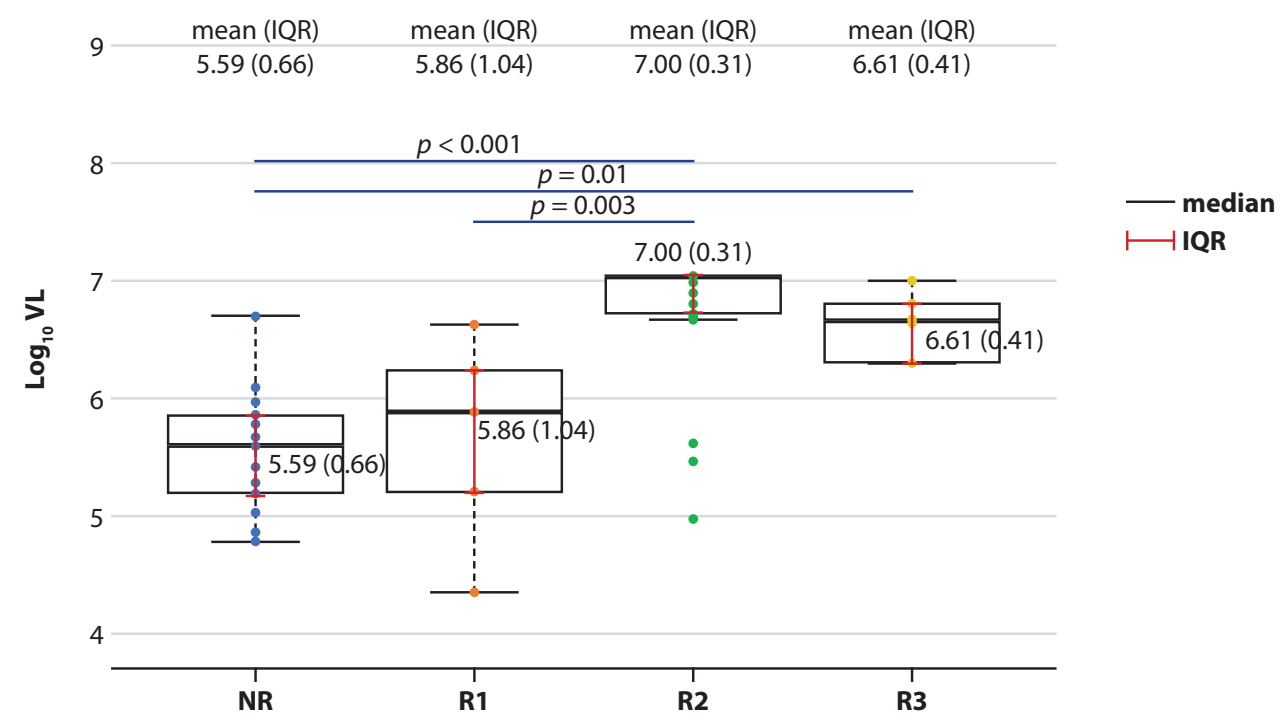

Figure 2. Boxed plot illustration of $\log _{10}$ VL with median (IQR) in NR (non-reactive), R1(antibody positive only), R2 (p24 antigen positive only) and $\mathrm{R} 3$ (both $\mathrm{p} 24$ antigen and antibody positive) 


\section{Correlation of HIV-RNA with Alere ${ }^{\mathrm{TN}} \mathrm{HIV}$ Combo results in acute HIV samples}

The median (IQR) $\log _{10}$ HIV-RNA between NR and $\mathrm{R}$ were 5.59 (5.19-5.85) and 6.86 (6.59-7.00) copies/mL, respectively $(p<0.001)$. Median (IQR) $\log _{10}$ HIV-RNA of R1, R2 and R3 were 5.86 (5.18-6.22), 7.00 (6.69-7.00) and 6.61 (6.266.75) copies/mL, respectively. $\mathrm{R} 2$ and $\mathrm{R} 3$ had significantly higher HIV-RNA than NR $(p<0.001$ and $p=0.01$, respectively). R2 also had significantly higher HIV-RNA than R1 ( $p$ $=0.003)$ (Table 1 and Figure 2).

\section{Discussion}

Our study, to the best of our knowledge, is the first study to look at the performance of Alere ${ }^{\mathrm{TM}}$ HIV Combo in a relatively large sample size of Thai AHI individuals infected mainly with HIV CRF01_AE subtype. ${ }^{19-20}$ Although Alere ${ }^{\text {TM }}$ HIV Combo could not detect $26 \%$ of AHI samples (NR) picked up by Abbott $4^{\text {th }}$ generation assay, it could diagnose $74 \%$ of $\mathrm{AHI}$ samples otherwise missed by $3^{\text {rd }}$ generation antibody testing alone. The samples that were missed by Alere ${ }^{\mathrm{Tn}}$ HIV Combo were most likely infected very early since they had much lower S/CO ratio on machine-based $4^{\text {th }}$ generation test and lowest HIV-RNA (Table 1, Figures 1 and 2). This again confirms previous study which shows that the sensitivity of Alere ${ }^{\mathrm{Tm}} \mathrm{HIV}$ Combo falls between machine-based $4^{\text {th }}$ generation test and $3^{\text {rd }}$ generation antibody test. ${ }^{15}$

The $74 \%$ sensitivity of Alere ${ }^{\mathrm{TN}}$ HIV Combo in detecting AHI in our study falls between its sensitivity found in the other two AHI studies from the Netherlands and United Kingdom of $65 \%$ and $88 \%$, respectively. ${ }^{21-22}$ However, these two studies used slightly different criteria for AHI from ours. Although HIV antibody was negative by $3^{\text {rd }}$ generation test in their studies as well as in our study, they used p24 antigen positivity to define AHI while we used machine-based $4^{\text {th }}$ generation positivity, following our routine testing algorithm, in order to allow practical implication of study results. $\mathrm{Ma}$ chine-based $4^{\text {th }}$ generation HIV Ag/Ab test cannot differentiate positivity for either antigen or antibody from positivity for both. However, it may not matter as long as we can diagnose an infected individual early. However, knowing someone with p24 antigen positivity, especially without concurrent antibody positivity, may be advantageous for epidemiological investigation and infection control, both for hot spot investigation and index testing since these are individuals with acute or recent infection who have greater potential in transmitting HIV to others. ${ }^{23-24}$ Alere $^{\mathrm{TM}}$ HIV Combo has this advantage.

Although our study did not have quantitative p24 antigen for direct comparison, our results demonstrated that the majority of Alere ${ }^{\mathrm{Tm}}$ HIV Combo positivity in our unique set of AHI plasma samples was due to p24 antigen positivity since there were $32(\mathrm{R} 2+\mathrm{R} 3) \mathrm{p} 24$ antigen positive samples and only $11(\mathrm{R} 1+\mathrm{R} 3)$ antibody positive samples (Table 1), a ratio of 2.9:1. This confirms other previous studies demonstrating that p24 antigen detection is essential in diagnosing AHI irrespective of the presence or absence of the HIV-specific antibody. ${ }^{25-27}$ From our study, Alere ${ }^{\text {TM }} \mathrm{HIV}$ Combo positivity in this set of AHI samples was correlated well with $\mathrm{S} / \mathrm{CO}$ ratios of the $4^{\text {th }}$ generation Abbott test, i.e., the higher the S/CO ratio, the more likely the positivity by Alere $^{\mathrm{Tm}}$ HIV Combo. Highest S/CO ratios were found in Alere $^{\mathrm{Tm}}$ HIV Combo p24 antigen positive samples (R2 + R3), which was also supported by higher HIV-RNA levels. This implies that machine-based $4^{\text {th }}$ generation assay also detects mainly p24 antigen in AHI samples. Furthermore, our HIVRNA results confirm previously published data showing optimal performance of Alere ${ }^{\text {TM }}$ HIV Combo in specimens that contained more than 10 million copies/mL of HIV-RNA. ${ }^{21}$

Our study is limited by the relatively small sample size of AHI but it is the first such study from the largest SEARCH 010/RV254 AHI cohort in Asia with the availability of detailed serologic and virologic data at diagnosis. Similar to all visually read test devices, it is subject to variable interpretation if the color line or spot is faint. The width of the Alere ${ }^{\text {tm }}$ HIV Combo strip is narrower than other strip devices such as Alere Determine HIV-1/2 strip, making it more difficult to interpret results with a shorter band. In addition, strip device of the test carries a higher chance of contamination due to specimen spillover as compared to cassette device.

\section{Conclusions}

We demonstrated that rapid Alere ${ }^{\mathrm{Tm}} \mathrm{HIV}$ Combo $4^{\text {th }}$ generation test, although less sensitive than machine-based $4^{\text {th }}$ generation test, could detect three quarters of AHI individuals missed by conventional $3^{\text {rd }}$ generation antibody tests. In a setting with high HIV incidence where machine-based $4^{\text {th }}$ generation test is not always available, rapid Alere ${ }^{\mathrm{mm}} \mathrm{HIV}$ Combo $4^{\text {th }}$ generation test is preferred as the first test in HIV testing algorithm over $3^{\text {rd }}$ generation antibody test. Once it is positive, it will then be further confirmed by the standard confirmatory testing algorithm or by HIV-RNA. Our study has the added value in using the S/CO ratio of machine-based $4^{\text {th }}$ generation antigen/antibody test and the HIV-RNA levels to give indirect evidence that Alere ${ }^{\mathrm{tm}}$ HIV Combo and machine-based $4^{\text {th }}$ generation test detect mainly p24 antigen in AHI.

\section{Acknowledgments}

The authors sincerely thank all participants and staff members at the Thai Red Cross Anonymous Clinic of the Thai Red Cross AIDS Research Centre. The Alere Combo kits were donated to the Thai Red Cross AIDS Research Centre for research purpose. We thank Gunn Pungpapong for his English language editing.

\section{References}

1. Brown AE, Mohammed H, Ogaz D, Kirwan PD, Yung M, Nash SG, et al. Fall in new HIV diagnoses among men who have sex with men (MSM) at selected London sexual health clinics since early 2015: testing or treatment or pre-exposure prophylaxis (PrEP)? Euro Surveill. 2017;22(25):30553.

2. Grulich A, Guy RJ, Amin J, Schmidt HM, Selvey C, Holden J, et al. Rapid reduction in HIV diagnoses after targeted PrEP implementation in NSW, Australia. Proceedings of Conference on Retroviruses and Opportunistic Infections; 2018 March 4-7; Boston, USA. Massachusetts; 2018.

3. San Francisco Department of Public Health. HIV Epidemiology Annual Report [Internet]. San Francisco; the HIV Epidemiology Section; 2016 [cited 2018 Mar 24]. Available from: https://www.sfdph.org/dph/files/ reports/RptsHIVAIDS/Annual-Report-2016-20170831.pdf.

4. Arora DR, Maheshwari M, Arora B. Rapid point-of-care testing for detection of HIV and clinical monitoring. ISRN AIDS. 2013;287269. 
5. Wongkanya R, Pankam T, Wolf S, Pattanachaiwit S, Jantarapakde J, Pengnongyang $\mathrm{S}$, et al. HIV rapid diagnostic testing by lay providers in a key population-led health service programme in Thailand. J Virus Erad. 2018;4:12-5.

6. Phanuphak N, Jantarapakde J, Himmad L, Sungsing T, Meksena R, Phomthong S, et al. Linkages to HIV confirmatory testing and antiretroviral therapy after online, supervised, HIV self-testing among Thai men who have sex with men and transgender women. J Int AIDS Soc. 2020; 23:e25448.

7. World Health Organization [Internet]. Geneva: World Health Organization; c2020 [cited 2020 Feb 1]. WHO list of prequalified in vitro diagnostic products; [about 12 screens]. Available from: www.who.int/ diagnostics_laboratory/evaluations/190404_prequalified_product_list.pdf

8. Department of Medical Science, National Institute of Health [Internet]. Nonthaburi: Department of Medical Science; c2019 [cited 2020 Feb 1]. Name list of manufacturer/importer of diagnostic kit for HIV infection [about 7 screens]. Available from: http://ttp.dmsc.moph.go.th/ttp/th/file/ file2download/2563/kit_evaluation/TestKitUpdateNov19.pdf

9. Cooper DA, Gold J, Maclean P, Donovan B, Finlayson R, Bames TG, et al. Acute AIDS retrovirus infection. Definition of a clinical illness associated with seroconversion. Lancet. 1985;1:537-40.

10. Rosenberg NE, Kamanga G, Phiri S, Nsona D, Pettifor A, Rutstein SE, et al. Detection of acute HIV infection: a field evaluation of the Determine HIV-1/2 Ag/Ab Combo test. J Infect Dis. 2012;205(4):528-34.

11. Ottiger $\mathrm{C}$ and Huber AR. Comparison of the new Alere ${ }^{\mathrm{Tn}} \mathrm{HIV}$ Combo with Alere Determine HIV-1/2 Ag/Ab Combo in acute primo and established HIV infections. Ann Clin Lab Res. 2015;3:22.

12. Fitzgerald N, Cross M, O'Shea S, Fox J. Diagnosing acute HIV infection at point of care: a retrospective analysis of the sensitivity and specificity of a fourth-generation point-of-care test for detection of HIV core protein p24. Sex Transm Infect. 2017;93:100-1.

13. Nakagiri I, Wada H, Tokunaga H, Fukuda H, Tasaka T, Sugihara T. Performance assessment of a newly developed rapid diagnostic reagent for human immunodeficiency virus. Kansenshogaku Zasshi. 2015;89:733-40. Japanese.

14. Tienen CV, Rugebregt S, Scherbeijn S, Gotz H, GeurtsvanKessel C. The performance of the Alere HIV combo point-of-care test on stored serum samples; useful for detection of early HIV-1 infections? Sex Transm Infect. 2018;94:331-3.

15. Livant E, Heaps A, Kelly C, Maharaj R, Samsunder N, Nhlangulela L, et al. The fourth generation Alere ${ }^{\mathrm{Tm}} \mathrm{HIV}$ Combo rapid test improves detection of acute infection in MTN-003 (VOICE) samples. J Clin Virol. 2017;94:15-21.
16. Pankam T, Saensiriphan S, Areeyolwattana S, Barisri N, Pengnonyang S, Sirivichayakul S, et al. The validation and evaluation of anti-HIV testing algorithm used in mobile clinic setting for men who have sex with men in metropolitan Bangkok, Thailand. Asian Pac J Allergy Immunol. 2018; 36:42-50.

17. Ananworanich J, Schuetz A, Vandergeeten C, Sereti I, de Souza M, Rerknimitr R, et al. Impact of multi-targeted antiretroviral treatment on gut $\mathrm{T}$ cell depletion and HIV reservoir seeding during acute HIV infection. PLoS One. 2012; 7:e33948.

18. Alere $^{\mathrm{TM}}$ HIV Combo (CE). Alere International Limited; 2017. Package Insert. 2017 Available from: alere.com/en/home/product-details/alere -hiv-combo.html. For Performance Evaluation Only 7D28 for evaluation ver.001

19. McCutchan FE, Hegerich PA, Brennan TP, Phanuphak P, Singharaj P, Jugsudee A, et al. Genetic variants of HIV-1 in Thailand. AIDS Res Hum Retroviruses. 1992;8:1887-95.

20. Ou Cy, Takebe Y, Weniger BG, Luo CC, Kalish ML, Auwanit W, et al Independent introduction of two major HIV-1 genotypes into distinct high risk populations in Thailand. Lancet. 1993;341:1171-4.

21. Faraoni S, Rocchetti A, Gotta F, Ruggiero T, Orofino G, Bonora S et al Evaluation of a rapid antigen and antibody combination test in acute HIV infection. J Clin Virol. 2013;57:84-7.

22. Smallwood M, Vijh R, Nauche B, Lebouché B, Joseph L, Pant Pai N Evaluation of a rapid point of care test for detecting acute and established HIV infection, and examining the role of study quality on diagnostic accuracy: a Bayesian meta-analysis. PLoS One. 2016;11:e0149592. Doi: 10.1371/journal.pone.0149592. eCollection 2016.

23. Campbell EM, Patala A, Li JF, Johnson JA, Westheimer E, Gay CL, et al. Phylodynamic analysis complements partner services by identifying acute and unreported HIV transmission. Viruses. 2020;12:E145.

24. Elliott T, Sanders EJ, Doherty M, Ndung'u T, Cohen M, Patel P, et al. Challenges of HIV diagnosis and management in the context of pre-exposure prophylaxis (PrEP), post-exposure prophylaxis (PEP) test and start and acute HIV infection: a scoping review. J Int AIDS Soc. 2019;22:e25419.

25. Eshleman SH, Khaki L, Laeyendecker O, Piwowar-Manning E, Johnson-Lewis L, Husnik M, et al. Detection of individuals with acute HIV-1 infection using the ARCHITECT HIV Ag/Ab Combo assay. J Acquir Immune Defic Syndr. 2009;52:121-4.

26. Daar ES, Pilcher CD, Hecht FM. Clinical presentation and diagnosis of primary HIV-1 infection. Curr Opin HIV AIDS. 2008; 3:10-5.

27. Cohen MS, Shaw GM, McMichael AJ, Haynes BF. Acute HIV-1 infection. N Engl J Med. 2011; 364:1943-54. 